Z Rheumatol 2010 $69: 579-580$

DOI 10.1007/s00393-009-0527-2

Online publiziert: 13. August 2010

(c) Springer-Verlag 2010

J. Wollenhaupt ${ }^{1}$ - G.-R. Burmester ${ }^{2}$

${ }^{1}$ Abteilung Rheumatologie und klinische Immunologie, Schön-Klinik Hamburg-Eilbeck, Hamburg

${ }^{2}$ Medizinische Klinik mit Schwerpunkt Rheumatologie und Klinische Immunologie, Charité-Universitätsmedizin Berlin

\title{
Non-TNF-Biologika in der Therapie
}

ckade der B-Lymphozyten-Aktivität und Inhibierung der T-Zell-Aktivierung - eröffnen sie weitere Therapieoptionen sowohl für Biologika-naive als auch für unzureichend auf eine TNF-Blockade ansprechende Patientengruppen. Erkrankungen aus einer ganzen Reihe von Substanzen wählen zu können. Diese bringen uns unserem Ziel näher, eine Remission oder zumindest eine niedrige Krankheitsaktivität auch bei chronischen Erkrankungen zu erreichen.

Dabei haben zweifelsohne die Biologika der ersten Generation, also die TNFBlocker, die Behandlung der chronischen Arthritiden und Spondylarthritiden revolutioniert. Mit ihnen wurde die Weiterentwicklung der Therapieziele möglich: Nicht mehr die die ausreichende Kontrolle der Entzündung und ihrer Auswirkungen auf Schmerz, Funktion und Knochenstruktur, sondern die (nahezu) vollständige Beherrschung der Entzündung, Freiheit von Arthritisschmerz und ein Stopp der radiologischen Gelenkzerstörung sind heute realistische Ziele geworden. In Frühfällen erscheint die frühzeitige Remission mit einer normalen Lebens- und Berufsperspektive für die $\mathrm{Pa}$ tienten erreichbar. Sowohl der effektivere Einsatz der konventionellen Basistherapie, vor allem Methotrexat, als auch Kombinationen von TNF-Blockern mit konventionellen Basistherapeutika erreichen diese Therapieziele bei vielen Patienten.

Vor diesem Hintergrund wurden in jüngster Vergangenheit weitere Biologika der zweiten Generation verfügbar (• Tab. 1).

Mit ihren differenzierten Ansätzen Inhibition anderer Zytokine als TNF, Blo-

\section{(7) Mit ihren differenzierten Ansätzen eröffnen Biologika weitere Therapieoptionen}

Weitere Biologika mit alternativen Ansatzpunkten werden erprobt und sehr wahrscheinlich als Biologika der 3. Generation unsere Behandlungsmöglichkeiten erneut erweitern (• Tab. 1).

Der Schwerpunkt dieser Ausgabe der Zeitschrift für Rheumatologie fasst den heutigen Kenntnisstand der einzelnen Substanzen, ihren derzeitigen Stellenwert im therapeutischen Algorithmus bei rheumatoider Arthritis und die sehr wahrscheinlich bald verfügbaren Optionen zusammen.

Deutlich kommt in den Beiträgen zum Ausdruck, wie differenziert und individuell die Therapie chronischer Arthritiden heute schon sein kann.

Die patientenindividuellen Therapieziele können dadurch noch besser definiert und konsequent verfolgt werden. Die Darstellung der einzelnen Substanzen durch Kollegen mit tiefgreifenden Kenntnissen der substanzspezifischen Daten sowie die Einordnung des Stellenwertes der Nicht-TNF-Biologika in der Therapiestrategie durch Wollenhaupt und Krüger sollen nicht vergessen lassen, dass viele $\mathrm{Pa}$ tienten durch Methotrexat, andere konventionelle Basistherapeutika und durch TNF-Blocker hervorragend behandelt werden können. Dennoch belegen die fol-

Tab. 1 Biologika der 2. und 3. Generation (so genannte „Non-TNF-Biologika“), die zur Behandlung der rheumatoiden Arthritis in Deutschland bereits zugelassen (kursiv) oder in der klinischen Prüfung (*mit Angabe der Prüfphase) sind

\begin{tabular}{|c|c|c|}
\hline Wirkprinzip & Ansatzpunkt & Substanz \\
\hline \multirow[t]{2}{*}{ Zytokinblockade } & $\begin{array}{l}\text { Interleukin-6 } \\
\text { Interleukin-17 }\end{array}$ & $\begin{array}{l}\text { Tocilizumab } \\
\text { Ly2439821 (*II, III) } \\
\text { AIN457 (*II, III) }\end{array}$ \\
\hline & Interleukin-22 & ILY-094 (*II) \\
\hline T-Zell-gerichtete-Kostimulation & CTLA-4 & Abatacept \\
\hline \multirow[t]{3}{*}{ B-Zell-gerichtete-Therapie } & $\mathrm{CD} 20$ & $\begin{array}{l}\text { Rituximab } \\
\text { Ofatumumab }\left({ }^{*} \mid \mathrm{II}\right) \\
\text { TRU-015 }\left({ }^{*} \mathrm{II}\right) \\
\left.\text { SBI-087 }{ }^{*} \mathrm{II}\right)\end{array}$ \\
\hline & BlyS & Belimumab $(* I I)$ \\
\hline & Blys+APRIL & Atacicept $\left({ }^{*}\right.$ II) \\
\hline Enzyminhibitoren der Zytokinaktivierung & $\begin{array}{l}\text { JAK } \\
\text { Syk } \\
\text { Phosphodiesterase }\end{array}$ & $\begin{array}{l}\text { Tasocitinib }\left({ }^{*} \mid I I\right) \\
\text { Fosamatinib-Diskodium (*II) } \\
\text { Apremilast }\left({ }^{*} \text { II) }\right.\end{array}$ \\
\hline
\end{tabular}


genden Beiträge unseres Themenschwerpunktes, wie chancenreich die Erweiterung des Therapiespektrums durch die Gruppe der Non-TNF-Biologika in der Rheumatologie heute geworden ist. Ihre

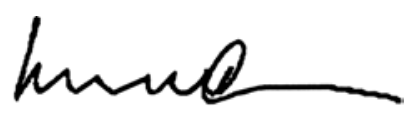

Jürgen Wollenhaupt

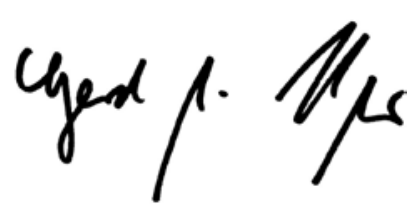

Gerd-R. Burmester

\section{Korrespondenzadresse}

Prof. Dr. J. Wollenhaupt

Abteilung Rheumatologie und klinische Immunologie, Schön-Klinik Hamburg-Eilbeck Dehnhaide 120, 22081 Hamburg wollenhaupt@rheumatologikum.de

\section{Eva-Luise-Köhler-Forschungs- preis für Seltene Erkrankungen 2011}

Die Eva-Luise-und-Horst-Köhler-Stiftung für Menschen mit seltenen Erkrankungen vergibt in Kooperation mit der Allianz Chronischer Seltener Erkrankungen (ACHSE e.V.) zum vierten Mal einen Preis für Forschungsprojekte, die sich seltenen Erkrankungen widmen. Der mit 50.000 Euro dotierte Forschungspreis ist nach der Schirmherrin der ACHSE, Frau Eva Luise Köhler, benannt.

Viele der über 5000 seltenen Erkrankungen bedeuten für den Patienten eine deutliche Verminderung der Lebensqualität und der Lebensdauer. Durch den Mangel an Forschung fehlen Medikamente und Therapien. Mit dem Eva-Luise-Köhler-Forschungspreis soll die Durchführung bzw. Anschubfinanzierung eines am Patientennutzen orientierten Forschungsprojektes im Bereich der seltenen Erkrankungen ermöglicht werden. Der Forschungspreis macht zudem Wissenschaft, Industrie und Gesellschaft darauf aufmerksam, dass die Forschung zu den vordringlichsten Aufgaben der Gesundheitspolitik gehören sollte. Bewerbungsschluss für den Forschungspreis ist der 04. Oktober 2010. Voraussichtlich am 28. Februar 2011 erfolgt dann in Berlin die feierliche Vergabe an den oder die Preisträger.

Interessierte Wissenschaftler erhalten detaillierte Informationen zur Bewerbung und die Bewerbungsunterlagen unter www.achse-online.de.

Quelle:

Allianz Chronischer Seltener Erkrankungen (ACHSE e. V.), www.achse-online.de
Fotowettbewerb „Bilder der Forschung" zum sechsten Mal ausgeschrieben

Zum sechsten Mal in Folge wurde mit „Bilder der Forschung" einer der größten deutschen Wettbewerbe für Wissenschaftsfotografie ausgeschrieben. Das Nachrichtenmagazin FOCUS und der Verband Forschender Arzneimittelhersteller e.V. (vfa), zeichnen Fotografen aus, deren Bilder die Faszination von Forschung visualisieren und das Interesse des Betrachters für Wissenschaft wecken. Bis zum 3. Oktober 2010 sind Wissenschaftler, professionelle Fotografen und ambitionierte Amateure aufgerufen, ihre spektakulärsten Bilder einzureichen. Gesucht werden Fotos aus Forschung und Wissenschaft sowie Porträts von Menschen, die in der Forschung arbeiten oder denen durch diese geholfen wird. Die beiden Kategorien „Faszination Forschung" und "Gesichter der Forschung" sind mit Preisgeldern von insgesamt 20.000 Euro dotiert. Eine prominent und fachkundig besetzte Jury wählt die Gewinner.

Ab Oktober kann zudem öffentlich auf FOCUS Online für den Gewinner des Publikumspreises abgestimmt werden. Alle Siegerfotos werden im Nachrichtenmagazin FOCUS, auf FOCUS Online und auf der Website des Wettbewerbs veröffentlicht. Die Gewinner erhalten ihre Auszeichnung im Rahmen einer Preisverleihung im Dezember 2010 in Berlin.

Nähere Informationen zu Ausschreibung, Teilnahmebedingungen und Anmeldeformulare unter:

www.bilder-der-forschung.de

Quelle: FOCUS Magazin Verlag GmbH, www.focus.de; Verband Forschender Arzneimittelhersteller e.V., www.vfa.de 